\title{
Evaluación de la langosta (Jasus frontalis) explotada en las islas Robinson Crusoe y Santa Clara del archipiélago de Juan Fernández (3340'S-8000'W)*
}

\author{
Eleuterio Yáñez $\mathbf{R}^{1}$., Cristian Canales $\mathbf{R}^{2}$ y Claudio Silva G. ${ }^{1}$ \\ ${ }^{1}$ Escuela de Ciencias del Mar, Universidad Católica de Valparaíso \\ Casilla 1020, Valparaíso, Chile \\ E-mail: eyanez@ucv.cl \\ ${ }^{2}$ Instituto de Fomento Pesquero, Casilla 8-V, Valparaíso, Chile \\ E-mail: ccanales@ifop.cl
}

Recibido: 15 diciembre 1998; versión corregida: 3 enero 2000; aceptado: 6 marzo 2000

\begin{abstract}
RESUMEN Se evalúa y analiza el estado de explotación del stock de la langosta (Jasus frontalis) explotada en las islas Robinson Crusoe y Santa Clara del archipiélago de Juan Fernández (3340'S-8000'W). Mediante el modelo de análisis de cohorte a la talla (ACT) ajustado a la pesquería realizada en la temporada 1996-97, se estiman los valores de mortalidad por pesca de los grupos completamente reclutados $\left(\mathrm{F}_{\mathrm{cr}}\right)$ en 0,63 para machos y 0,81 para hembras, con una talla al 50\% de retención de 94 a 96 para machos y 96 para hembras. Se estima una biomasa media de machos entre 29 y 31 toneladas (46.663 y 50.355 individuos respectivamente) y entre 39 y 42 toneladas (59.785 y 63.249 individuos respectivamente) para hembras. Por otra parte, en el análisis del rendimiento equilibrado se estima que las mortalidades por pesca $\mathrm{F}_{\text {cr }}$ derivadas del modelo ACT, resultaron inferiores al valor recomendado $\mathrm{F}_{\text {máx }}$ estimado en 0,86 en machos y 1,15 en hembras, y superiores a un nivel de mortalidad $\mathrm{F}_{0.1}$. El análisis de rendimiento equilibrado de largo plazo (CBA), sugiere para una estrategia de explotación $\mathrm{F}_{0.1}$, que el valor recomendado de desembarque equivale a 10,4 y 14,6 toneladas (16.885 y 15.449 individuos) de machos y hembras respectivamente, cifras inferiores en un $10 \%$ respecto a los actuales niveles desembarcados. La talla crítica de langosta para un valor de $\mathrm{M}=0,18$, se determinó igual a $110 \mathrm{~mm}$ en machos y $107 \mathrm{~mm}$ en hembras, mientras la edad crítica estimada para ambos sexos corresponde al grupo de edad de 9 años. Se concluye que la situación actual de la langosta de Juan Fernández estaría implicando intensos niveles de explotación.
\end{abstract}

Palabras claves: evaluación, stock, langosta, archipiélago de Juan Fernández, Chile.

\section{Assessment of the spiny rock lobster (Jasus frontalis) exploited in the Robinson Crusoe and Santa Clara islands of Juan Fernandez archipelago $\left(3^{\circ}{ }^{\circ} 40^{\prime} \mathrm{S}^{-80^{\circ} 00^{\prime} \mathrm{W}}\right)^{*}$}

\begin{abstract}
ABSIRACT. The spiny rock lobster (Jasus frontalis) stock exploited in the Robinson Crusoe and Santa Clara islands

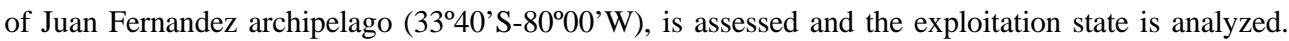

An analysis of cohort at size (ACT) model is adjusted to the fishery carried out in the 1996-97 season. The fishing mortality of the totally recruited groups $\left(\mathrm{F}_{\mathrm{cr}}\right)$ are estimated in 0,63 for males and 0,81 for females, with a $50 \%$ of retention size of 94 to 96 for males and 96 for females. The lobsters mean biomass is estimated in 29 to 31 ton (46.663 and 50.355 individuals respectively) for males and in 39 to 42 ton (59.785 and 63.249 individuals respectively) for
\end{abstract}

\footnotetext{
Artículo generado como parte del proyecto "Análisis y evaluación indirecta de la pesquería de la langosta en el archipiélago de Juan Fernández” (Proyecto FIP 96/22), financiado por el Fondo de Investigación Pesquera y ejecutado por la Universidad Católica de Valparaíso.
} 
females. On the other hand, in the equilibrated yield analysis is estimated that fishing mortality $\mathrm{F}_{\mathrm{cr}}$ derived of the ACT model, was inferior to the $\mathrm{F}_{\text {max }}$ recommended value estimated in 0,86 for males and 1,15 for females, and superiors to the $\mathrm{F}_{0.1}$ mortality. The long term equilibrated yield analysis $(\mathrm{CBA})$, suggests for a $\mathrm{F}_{0.1}$ exploitation strategy that the recommended landing value is equal to 10,4 and 14,6 ton (16.885 and 15.449 individuals) of males and females respectively, $10 \%$ inferior to current landings levels. The critical size of lobster for a value of $\mathrm{M}=0,18$, was determined similar to $110 \mathrm{~mm}$ in males and $107 \mathrm{~mm}$ in females, while the calculated critical age for both sexes corresponds to the group of 9 year-old age.

The lobster state situation indicates that the current exploitation levels are intense and with exploitation rates that would reduce the mean biomass in the long term. The biomass would reduce the yearly total levels to 50 ton (males + females), significantly inferior magnitude if an exploitation rate equivalent to $F_{0.1}$ biological point will be implemented, which would reach a yearly population equivalent size of 70 ton.

Key words: assessment, stock, spiny rock lobster, Juan Fernandez archipelago, Chile.

\section{INTRODUCCIÓN}

La langosta (Jasus frontalis) desde un punto de vista comercial, es la principal especie de la pesquería de crustáceos en el archipiélago de Juan Fernández, constituido por las islas Robinson Crusoe, Santa Clara y Alejandro Selkirk $\left(33^{\circ} 40^{\prime} \mathrm{S}-80^{\circ} 00^{\prime} \mathrm{W}\right)$. El stock es explotado por pescadores artesanales utilizando trampas construidas con madera y embarcaciones tipo botes balleneros de doble proa (8-10 $\mathrm{m}$ de eslora).

En la evolución de los desembarques de la pesquería de Jasus frontalis realizados en el archipiélago de Juan Fernández e islas Desventuradas se puede destacar que en 1938, 1942 y en el período 1952-1964, se realizaron desembarques de 120 t/año y aún superiores (Fig. 1). Desde ese último año, hasta la temporada de pesca 1979/ 80 , la tendencia fue sostenida, disminuyendo las captu-

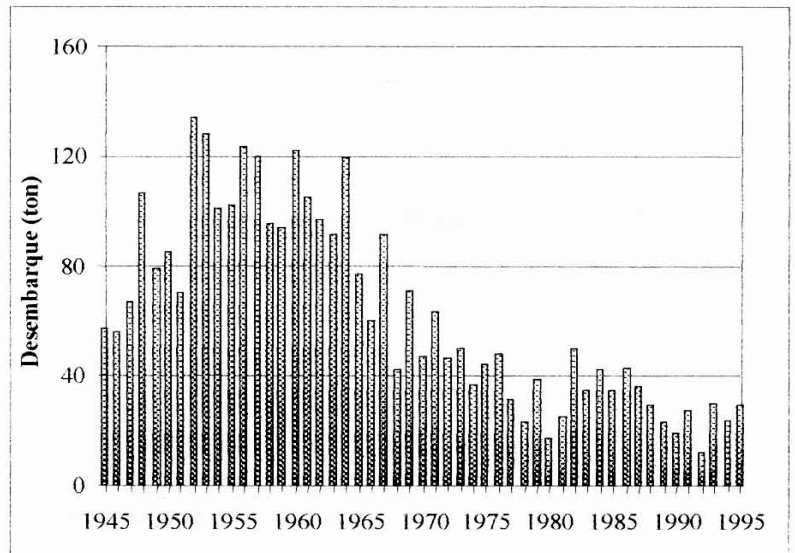

Figura 1. Desembarque anual de la langosta de Juan Fernández (Sernapesca, 1938-97).

Figure 1. Anual landings of the Juan Fernandez spiny rock lobster. ras hasta un décimo del nivel de esa época. Durante el período 1981-1986 las capturas aumentaron levemente y luego han disminuido progresivamente, alcanzando en 1997 un desembarque de 32 toneladas.

Una posible explicación para la situación deprimida en que se encuentra la pesquería de la langosta de Juan Fernández, estaría dada por el incremento excesivo del esfuerzo pesquero observado tanto en número de trampas caladas como en la eficiencia de ellas. A comienzos de la década del 80 se mejoró la efectividad de estos aparejos al ubicar la entrada en la parte superior, trampas "tipo L" a diferencia del diseño anterior, "tipo I", en que los túneles estaban en línea (Arana, 1983).

A comienzos de la década del 80 se realizaron estudios de evaluación del stock de langosta aplicando modelos globales de producción, se estableció que de acuerdo al tamaño del stock de langostas, el esfuerzo que se ejercía hasta esa fecha estaba sobredimensionado en más de un $30 \%$ y que de persistir dicha situación, podría afectar seriamente a la producción natural del mismo (Larraín, 1981; Yáñez et al., 1982, 1985). Como medida de protección del recurso se propuso establecer cuotas de pesca para la pesquería de las islas Robinson Crusoe y Santa Clara, como parte de una cuota total para la pesquería desarrollada en todo el archipiélago (Yáñez et al., 1985).

Considerando la información recopilada durante la temporada de pesca 1996-97, se evalúa en forma indirecta y se analiza el estado de explotación del stock de langosta en las 
islas Robinson Crusoe y Santa Clara del archipiélago de Juan Fernández. Para la evaluación del stock se propone el modelo de análisis de cohorte a la talla (ACT), basado en el análisis de pseudo-cohortes que requiere de datos de un sólo año. Para analizar el estado de explotación del stock, se estiman los puntos biológicos de referencia aplicando el modelo de rendimiento equilibrado de Thompson y Bell (1934), se calculan la tasa de explotación y excedentes productivos. Se realizó además, un análisis de sensibilidad del modelo de evaluación ante variaciones de los parámetros del vector de mortalidad por pesca.

\section{MATERIALES Y MÉTODOS}

\section{Método de evaluación del stock estructurado a la talla}

Ante la ausencia de una secuencia de estructuras de tallas históricas de los desembarques, se evaluó el stock presente durante la temporada 1996-1997 basándose en el modelo "Análisis de pseudo-cohorte a la talla" (Jones, 1984), para lo cual se acepta la hipótesis que el stock se encuentra en condiciones de equilibrio y que los reclutamientos y mortalidad a la talla han sido constantes en los últimos años. Cabe señalar que este mismo enfoque fue considerado para la pesquería de langosta de Juan Fernández por Yáñez et al. (1985).

Se evalúa el stock de langosta estructurado a la talla y por sexo, para ello se considera la información recolectada en la temporada 1996-1997, de captura total estructurada a la talla y por sexo. Se propone el modelo de Análisis de Cohortes a la Talla (ACT), basado en el modelo de "pseudo-cohorte" de Jones (1984) considerando las siguientes modificaciones: patrón selectivo talla-específico (Lai y Amjoun, 1993); estimación no lineal mediante el empleo de una subrutina de optimización de acuerdo a Zuleta y Moreno (1993) y Canales (1994); y ecuación recursiva de MacCall (1986) a la talla.

En general, para la estimación de biomasa y abundancia por intervalo de talla para el stock de langosta para machos y hembras, se consideraron los siguientes supuestos: la tasa de mortalidad natural permanece constante entre los intervalos de talla y durante el período analizado (temporada 19961997); la función de crecimiento se basa en el modelo de von Bertalanffy (1938); el patrón de reclutamiento (o selectividad) a la pesquería está defini- do por una función de tipo logística; y la estructura de tallas de la captura es representativa de una situación de un estado equilibrado.

Para el cálculo del número de sobrevivientes $\left(\mathrm{N}\left(\mathrm{L}_{\mathrm{i}}\right)\right)$ que en un determinado período de tiempo (dt), lograron llegar al intervalo de tallas i-ésimo, se empleó la ecuación recursiva propuesta por Pope (1972) y modificada por MacCall (1986):

$$
N\left(L_{i}\right)=N\left(L_{i+1}\right) * e^{M * \delta_{t_{i}}}+\frac{C\left(L_{i}\right)_{o b s} * M * \delta t_{i}}{1-e^{-M^{*} \delta_{i}}}
$$

donde $\mathrm{M}$ es la mortalidad natural de entrada y $C\left(L_{i}\right)_{\text {obs }}$ corresponde a la captura observada en número para el i-ésimo intervalo de tallas, la que corresponde a la siguiente función:

$$
C\left(L_{i}\right)_{o b s}=C\left(L_{i}\right)_{c o m}+C\left(L_{i}\right)_{c a s}
$$

donde $C\left(L_{\mathrm{i}}\right)_{\text {com }}$ es la frecuencia de individuos tomados del desembarque comercial correspondiente al intervalo de tallas i-ésimo y $\mathrm{C}\left(\mathrm{L}_{\mathrm{i}}\right)_{\text {cas }}$ es el número de individuos del intervalo obtenido de la expansión de la estructura de tallas al desembarque estimado como utilizado para consumo local de individuos bajo talla mínima legal. El tiempo requerido por un individuo en crecer desde el intervalo de tallas $i$ al intervalo $i+1$ se obtiene con la expresión:

$$
\delta t_{i}=\frac{1}{k} * \ln \left(\frac{L_{\infty}-L_{i}}{L_{\infty}-L_{i+1}}\right)
$$

donde $\mathrm{L}_{\infty}$ y k corresponden a los parámetros de la función de crecimiento langosta.

Por otro lado, el valor de mortalidad natural (M) de entrada al modelo corresponde a aquel valor que sea estimado de los modelos bio-analógicos y tallaestructurados (ver más adelante). Debido a la incertidumbre de dicho parámetro, se sensibilizan las salidas del modelo a valores mínimo, medio y máximo de este parámetro.

La abundancia de partida correspondiente al último intervalo de tallas (1), se obtiene al ser despejada la ecuación de captura de Baranov (1918) de la forma:

$$
N\left(L_{\lambda}\right)=\frac{C\left(L_{\lambda}\right)^{*}\left(F\left(L_{\lambda}\right)+M\right)}{F\left(L_{\lambda}\right) *\left(1-e^{-\left(\left(F\left(L_{\lambda}\right)+M\right)^{*} \dot{\delta}_{i}\right)}\right)}
$$


donde $\mathrm{F}\left(\mathrm{L}_{1}\right)$ corresponde a la mortalidad por pesca del último grupo de tallas. La mortalidad por pesca $\left(F\left(L_{i}\right)\right)$ de entrada al modelo, se descompone en dos efectos: uno asociado a la mortalidad por pesca de los grupos completamente reclutados $\left(\mathrm{F}_{\mathrm{cr}}\right)$ y otra, a la selectividad que presentan las trampas empleadas en la captura de la langosta $\left(\mathrm{S}\left(\mathrm{L}_{\mathrm{i}}\right)\right)$ :

$$
F\left(L_{i}\right)=F_{c r} * S\left(L_{i}\right)
$$

Ante el desconocimiento que presenta el patrón de reclutamiento, se propone la función de selectividad (modelo logístico generalizado):

$$
S\left(L_{i}\right)=\frac{1}{1+e^{a-b^{*} \bar{L}^{i}}}
$$

donde a, b y c son los parámetros de la función de selectividad y $\overline{L_{i}}$ es la longitud media del intervalo de tallas i-ésimo.

De esta forma la distribución de tallas de las capturas son predichas mediante la expresión:

$$
C\left(L_{i}\right)_{\text {pred }}=\frac{F\left(L_{i}\right)^{*} N\left(L_{i}\right) *\left(1-e^{-Z\left(L_{i}\right)^{*} \dot{\alpha}_{i}}\right)}{Z\left(L_{i}\right)}
$$

determinando para tal el efecto los parámetros $q=\left[F_{c r}\right.$ a b], que minimicen la función negativa log-verosímil condensada:

$-\log (L($ datos $/ \theta))=\frac{1}{N} * \ln \left(\sum_{i=1}^{\lambda}\left\{\ln \left(C\left(L_{i}\right)_{\text {pred }}\right)-\ln \left(C\left(L_{i}\right)_{\text {obs }}\right)\right\}^{2}\right)$

Una vez resuelta la función, los errores estándar de cada uno de los parámetros del modelo, así como de las biomasa y abundancias, se calcularon a través de inferencia bayesiana, suponiendo distribuciones a priori no informativas (uniformes) de los parámetros.

La teoría de bayes indica que una medida de la credibilidad de un cierto conjunto de parámetros (q,) está dada por una probabilidad conjunta a posteriori resultante del producto normalizado entre la probabilidad conjunta a priori de los parámetros, y el desempeño del experimento dado el conjunto de parámetros impuesto. El teorema se resume en la clásica expresión:

$$
P\left(\theta_{i} / \text { datos }\right)=\frac{P\left(\theta_{i}\right) L\left(\text { datos } / \theta_{i}\right) d \theta}{\int P(\theta) L(\text { datos } / \theta) d \theta}
$$

donde el subíndice $\mathrm{i}$ indexa a una única combinación de parámetros del vector q.

El método de cálculo empleado consistió en establecer una grilla en el hiperespacio, como resultado de establecer n-intervalos dentro de un rango de valores probables de cada uno de los parámetros. De este modo, en cada una de las celdas (combinaciones) se evaluó el kernel posterior como una medida proporcional a la distribución de probabilidad posterior conjunta.

$$
P\left(\theta_{i} / \text { datos }\right) \propto P\left(\theta_{i}\right) L\left(\text { datos } / \theta_{i}\right)
$$

donde la distribución a priori conjunta, en este caso, equivale a:

$$
P\left(\theta_{i}\right)=P\left(F_{c r, i}\right) P(a, i) P(b, i)
$$

Seguidamente, ya evaluadas todas las combinaciones, la distribución de probabilidad posterior conjunta se construyó en términos discretos como:

$$
P\left(\theta_{i} / \text { datos }\right)=\frac{P\left(\theta_{i}\right) L\left(\text { datos } / \theta_{i}\right)}{\sum_{i=1}^{m} P\left(\theta_{i}\right) L\left(\text { datos } / \theta_{i}\right)}
$$

donde $\mathrm{m}$ corresponde al total de combinaciones.

Finalmente, las distribuciones de probabilidad marginales posteriores, tanto de los parámetros como de las biomasas, se construyen integrando sobre la conjunta en la forma:

$$
P\left(\theta_{j} / \text { datos }\right)=P\left(\theta_{j, n} \leq \theta_{j}<\theta_{j, n+1}\right)=\frac{\sum_{i=1}^{m} H_{i, j} P\left(\theta_{i}\right) L\left(\text { datos } / \theta_{i}\right)}{\sum_{i=1}^{m} P\left(\theta_{i}\right) L\left(\text { datos } / \theta_{i}\right)}
$$

donde $j$ caracteriza a un parámetro específico (por ej. For) o bien la biomasa, $n$ mide la amplitud del intervalo y $\mathrm{H}_{\mathrm{i}, \mathrm{j}}=1$ si $q_{\mathrm{j}, n} £ q_{\mathrm{j}}<q_{\mathrm{j}, n+1} \mathrm{yH}_{\mathrm{i}, \mathrm{j}}=0$ en caso contrario.

Para estos efectos y a la luz de los resultados de la evaluación de stock, la abundancia y biomasa media de langosta son estimados por rango de talla y sexo mediante la expresión:

$$
\begin{gathered}
\bar{N}\left(L_{i}\right)=\frac{N\left(L_{i}\right)-N\left(L_{i+1}\right)}{Z\left(L_{i}\right)} \\
\bar{B}\left(L_{i}\right)=\frac{N\left(L_{i}\right) * W\left(L_{i}\right)-N\left(L_{i+1}\right) * W\left(L_{i}\right)}{Z\left(L_{i}\right)}
\end{gathered}
$$

donde $\mathrm{W}\left(\mathrm{L}_{\mathrm{i}}\right)$ corresponde al peso medio corporal por 
grupo de talla, y es calculado a partir de los parámetros somatométricos a y b obtenidos de las relaciones longitud-peso por sexo de langosta establecidas, mediante la siguiente ecuación:

$$
W\left(L_{i}\right)=a^{*}\left(\frac{L+L_{i+1}}{2}\right)^{b}
$$

De esta manera, la población media total de langostas se determina como la suma de las poblaciones media (abundancia y biomasa) de la forma:

$$
\bar{N}=\sum_{i=1}^{\lambda} \bar{N}\left(L_{i}\right) \quad \bar{B}=\sum_{i=1}^{\lambda} \bar{B}\left(L_{i}\right)
$$

donde los intervalos de confianza de los estimadores serán precisados a través de simulación montecarlo incorporando para ello, el error estándar de los parámetros resueltos por el modelo. Es decir, se realizan tantas evaluaciones de stock como número de réplicas de la frecuencia de talla se generen, considerándose para este efecto un número cercano a 1.000 .

Las mortalidades por pesca evaluadas para el stock de langosta, son las siguientes:

- Mortalidad por pesca de grupos completamente reclutados $\left(\mathrm{F}_{\mathrm{cr}}\right)$ : esta mortalidad por pesca proviene de la evaluación de stock descrita anteriormente, la cual sirve de comparación respecto de mortalidades por pesca de referencia $\left(\mathrm{F}_{0.1}\right.$ y $\left.\mathrm{F}_{\text {máx }}\right)$ para definir el estado de explotación de la langosta.

- Mortalidad por pesca global $\left(\mathrm{F}_{\text {glob }}\right)$ : no considera situaciones estructurales y describe la mortalidad por pesca total del stock. Para estos efectos y considerando que la abundancia estimada corresponde a la fracción remanente a la mitad de la temporada extractiva, la mortalidad por pesca global se determina por:

$$
F_{g l o b}=\frac{C}{\bar{N}}
$$

- Mortalidades por pesca de referencia $\left(\mathrm{F}_{0.1} \mathrm{y}\right.$ $\left.\mathrm{F}_{\text {máx }}\right)$ : Los resultados de la evaluación de stock, la relación longitud-peso y el vector de selectividad a la talla $\left(\mathrm{S}\left(\mathrm{L}_{\mathrm{i}}\right)\right)$, permiten la aplicación de un modelo de rendimiento estructurado como el de Thompson y Bell
(1934), con el cual son estimados los puntos biológicos de referencia $\mathrm{F}_{0.1}$ y $\mathrm{F}_{\text {máx }}$.

- Mortalidad por pesca total $\left(\mathrm{Z}_{\text {glob }}\right)$ : la mortalidad total del stock de langosta es determinada por la suma:

$$
Z_{\text {glob }}=F_{g l o b}+M
$$

\section{Datos de entrada}

En la aplicación del modelo ACT se considera como datos de entrada la información estadística recopilada en el proyecto FIP N N $^{\circ}$-22: "Análisis y evaluación indirecta de la pesquería de langosta en el archipiélago de Juan Fernández”. Basándose en los datos obtenidos de muestreos específicos de las capturas realizadas en las islas Robinson Crusoe y Santa Clara durante la temporada de pesca 1996-97, se construyen las estructuras de tallas del desembarque comercial y a lo que se ha denominado como "consumo casero" de los habitantes de las islas (Arana y Vega, 2000).

Se requiere además, de los siguientes parámetros biológicos de la especie (Tabla 1): relación longitud-peso por sexo, debido a la significativa diferencia entre machos y hembras; función de crecimiento por sexo basado en el modelo de von Bertalanffy; mortalidad natural promedio calculada utilizando las fórmulas de Taylor (1958) y Pauly (1980), evaluadas con diferentes valores de $\mathrm{M}$ (valor estimado \pm 0.02 ) para comparar los resultados del método utilizado.

\section{Puntos biológicos de referencia}

Este punto deriva de la estimación de las mortalidades por pesca globales de referencia del punto anterior, $\mathbf{F}_{\mathbf{0 . 1}}, \mathbf{F}_{\text {máx }}$ y $\mathbf{F}_{\mathbf{c}}$, donde esta última permite evaluar el actual estado de explotación del recurso, al ser comparada con mortalidades por pesca referenciales derivadas del modelo de rendimiento equilibrado de Thompson y Bell (1934) estructurado a la talla. Dicho modelo, se fundamenta en suponer una condición ideal de equilibrio, requiriendo un estimado del reclutamiento $(\mathrm{R})$ y del vector patrón de selectividad (r) obtenidos por el modelo ACT. De esta forma, se obtiene para cada nivel de mortalidad por pesca, un determinado valor de rendimiento equilibrado de largo plazo de acuerdo a la expresión:

$$
Y(F)=\sum_{i=1}^{\lambda} \frac{F^{*} r\left(L_{i}\right) *\left(1-e^{-Z\left(L_{i}\right) * \delta_{i}}\right)}{Z\left(L_{i}\right)} * N\left(L_{i}\right) * \bar{W}\left(L_{i}\right)
$$


donde la abundancia en cada intervalo de talla, se evalúa por la expresión:

$$
N\left(L_{i}\right)=R * e^{\left(-\sum_{i=1}^{\lambda} Z\left(L_{i}\right) * \delta t_{i}\right)}
$$

la curva de biomasa media equilibrada se determina por:

$$
\bar{B}(F)=\frac{1}{F} * Y(F)
$$

\section{Tasa de explotación y excedentes productivos}

La tasa de explotación entendida como la fracción de la población removida por efecto de la pesca se determina con la expresión:

$$
\mu=\frac{F_{g l o b} *\left(1-e^{\left(-F_{g l b b}-M\right)}\right)}{F_{g l o b}+M}
$$

La estimación de los excedentes productivos del recurso en cuestión, se determinan a través de una Captura Biológica Adecuada (CBA), evaluada en los distintos valores de mortalidad por pesca de referencia. Su expresión general corresponde a :

$$
C B A\left(F_{r e f}\right)=\sum_{i=1}^{\lambda} \frac{F_{r e f} * r\left(L_{i}\right) *\left(1-e^{-Z\left(L_{i}\right) * \delta_{i}}\right)}{Z\left(L_{i}\right)} * N\left(L_{i}\right) * \bar{W}\left(L_{i}\right)
$$

donde $F_{r e f}$ corresponde al nivel de mortalidad por pesca recomendable que en este caso particular, corresponderán a los niveles $\mathrm{CBA}\left(\mathrm{F}_{0.1}\right)$ y $\mathrm{CBA}\left(\mathrm{F}_{\text {max }}\right)$.

\section{Estado de explotación del stock}

Con el fin de evaluar el estado de explotación del stock de langosta de Juan Fernández, se emplearon los siguientes criterios:

Tabla 1. Parámetros biológicos de Jasus frontalis.

Table 1. Biological parameters of Jasus frontalis.

\begin{tabular}{|lrcc|}
\hline $\begin{array}{l}\text { Parámetro } \\
\text { Biológico }\end{array}$ & Hembras & Machos & Autor \\
\hline $\begin{array}{lrr}\text { Crecimiento } \\
\text { Loo (mm) }\end{array}$ & 214,413 & 212,578 & Arana y Olate, 2000 \\
k & 0,0697 & 0,0707 \\
\multicolumn{4}{|c}{} \\
Relación Longitud / Peso & \\
A & 0,0034 & 0,0017 & Arana y Olate, 2000 \\
B & 2,6597 & 2,7830 \\
\hline
\end{tabular}

1. Análisis de mortalidades por pesca: en este sentido, se evalúa la magnitud de la mortalidad por pesca de los grupos de tallas completamente reclutados a la pesquería $\left(\mathrm{F}_{\mathrm{cr}}\right)$ respecto a las mortalidades por pesca biológicas derivadas del análisis de rendimiento equilibrado, empleando los siguientes criterios:

a) si $_{\text {cr }}>F_{\text {máx }}$, el stock se encuentra en estado de sobrepesca por crecimiento.

b) si $\mathrm{F}_{0,1}<\mathrm{F}_{\mathrm{cr}}<\mathrm{F}_{\text {máx }}$, el stock se encuentra en plena o fuerte explotación.

c) $\mathrm{siF}_{\mathrm{cr}}<\mathrm{F}_{0,1}$, el stock se encuentra sub-explotado y permite un mayor nivel de extracción.

2. Análisis de la modificación de la talla de primera captura: Como la pesquería de langosta reviste vital importancia en la fuente de ingresos del archipiélago de Juan Fernández, se propone un análisis para evaluar la conveniencia de modificar la talla de primera captura. En este sentido se evaluó la eficiencia de la pesquería en relación al crecimiento somático del recurso, determinándose para dicho efecto la talla crítica $\left(L_{\text {crit }}\right)$ en la cual la razón de cambio entre la biomasa afectada sólo por pérdidas debido a causas naturales y la talla se hace cero:

$$
\frac{d B}{d t}=\frac{d *\left(N_{0} * e^{M t} * W_{\infty} *\left(1-e^{k^{*}\left(t-t_{o}\right)}\right)^{b}\right)}{d t}=0
$$

lo que conduce a la expresión de edad crítica como:

$$
t_{c r i t}=t_{o}-\frac{1}{k} * \ln \left(\frac{M}{M+b^{* k}}\right)
$$

y a la talla crítica de la forma:

$$
L_{c r i t}=\frac{b^{*} k^{*} L_{\infty}}{M+b^{*} k}
$$

De esta forma y una vez conocido el valor de $\mathrm{L}_{\text {crit }}$, se prueban distintas tallas de primera captura inferiores a la talla mínima legal de extracción vigente a través de curvas de rendimiento por recluta independientes de la selectividad de las trampas.

En este contexto, se propone el modelo de Beverton y Holt (1957) talla-estructurado, de la forma:

$$
\frac{y}{r}=F_{i} * A^{*} W_{\infty} *\left(\frac{1}{z}-\frac{3 * u}{z+k}+\frac{3 * u^{2}}{z+2 * k}-\frac{u^{3}}{z+3 * k}\right)
$$


donde

$$
u=1-\frac{L_{c}}{L_{\infty}} A=\left(\frac{L_{\infty}-L_{c}}{L_{\infty}-L_{r}}\right)^{\frac{m}{k}}
$$

siendo $\mathrm{L}_{\mathrm{c}}$ la talla de primera captura y $\mathrm{L}_{\mathrm{r}}$ la talla de reclutamiento al área de pesca (al 50\%), estimada como:

$$
L_{r}=\sum_{L=L_{1}}^{L_{\lambda}} L^{*} C(L) / \sum_{L=L 1}^{L_{\lambda}} C(L)
$$

\section{Variabilidad de los estimadores poblacionales}

Con el fin de medir el impacto en los tamaños poblacionales y las Capturas Biológicas Adecuadas (CBA) derivadas del modelo de evaluación, se realizó un análisis de sensibilidad de dicho modelo ante variaciones de los parámetros del vector de mortalidad por pesca. Una vez determinado los valores y los respectivos errores estándares de los parámetros del vector de mortalidad por pesca, se asume que cada valor puede ser representado por una función de probabilidad normal acotada entre los límites de confianza al $95 \%$, con media $\overline{\mathrm{C}}$ y desviación estándar $s$ conocidas, esto permite la obtención de resultados en condiciones medias y extremas.

\section{RESULTADOS Y DISCUSIÓN}

\section{Evaluación indirecta del stock de langosta}

Se aplicó el modelo ACT a la estructura de tallas de los desembarques del período 1996-97, para un valor central de mortalidad natural equivalente a $\mathrm{M}=0,18$. Los parámetros que determinaron el vector de mortalidades por pesca a la talla $\left(\mathrm{a}, \mathrm{b}, \mathrm{c} \mathrm{y} \mathrm{F}_{\mathrm{cr}}\right)$ y sus correspondientes errores estándar se presentan en la Tabla 2.

Se observa que el valor de la mortalidad por pesca de los grupos completamente reclutados a la pesquería de langosta, alcanzó en el período 1996-1997, un valor en machos de 0,63 anual, en tanto que en hembras este parámetro alcanzó un mayor valor estimado ( 0,81 anual), valores de mortalidad que se logran cuando el patrón de explotación llega a un $100 \%$. En efecto, se observa que el vector de selectividad talla-específico en machos y hembras alcanza su máxima pendiente en el rango de 93 y $99 \mathrm{~mm}$ de longitud cefalotoráxica, y que sobre estas tallas, el valor de la selectividad alcanza el 100\% (Fig. 2). De igual manera, se destaca que la talla al $50 \%$ de retención se alcanza en machos entre los 94 y 96 $\mathrm{mm}$, en tanto que en las hembras se logra a los 96 $\mathrm{mm}$, situación que revela un comportamiento abrupto del patrón de explotación, particularmente en torno a la vecindad acotada por el rango donde la pendiente alcanza su máxima expresión.

Las capturas a la talla predichas por el modelo se ajustan, en general, satisfactoriamente dentro de todo el espectro de tallas analizado, destacándose en hembras, una ligera subestimación de las primeras clases de tallas y de la moda observada (Fig. 2).

Los resultados de la evaluación del stock se presentan en la Tabla 3, en la cual se indica el nivel de desembarque promedio registrado, y las biomasas y abundancias medias totales y explotables que sustentaron estos desembarques, con tres valores probables de mortalidad natural. Se debe mencionar que del desembarque total registrado en ambos sexos $(27,8 \mathrm{t}), 2,6$ ton corresponden a la captura para consumo local de machos, y 3,3 ton de hembras, ambos registros referidos a individuos comprendidos entre 86 y $97 \mathrm{~mm}$ de longitud cefalotorácica.

La biomasa media de machos se estima habría alcanzado entre 1996 y 1997 valores entre 28,7 y 30,9 ton, de las cuales, cerca del $50 \%$ se encontraría constituida por ejemplares sobre la talla mínima legal de extracción (98 mm). De igual forma, en hembras los valores de biomasa oscilarían entre 39,4 y 41,7 ton, de las cuales cerca del $43 \%$ se encontrarían sobre la talla mínima de captura.

Estos niveles de biomasa se traducen en un efectivo poblacional equivalente a los rangos: 46,6-50,3 mil machos y 59,7-63,2 mil hembras, de los cuales en general, para un valor de $\mathrm{M}=0,18$, cerca de 40 mil individuos se encontrarían en condiciones de ser explotados (machos + hembras), correspondientes a 37,8 ton totales de ambos sexos.

Por otro lado, se destaca que durante este período de tiempo, las tasas de mortalidad por pesca global ponderada por el número de individuo capturado alcanzaron valores entre 0,31 y 0,33 en machos, en tanto que en hembras fluctuó entre 0,32 y 0,34 anual. Esta situación habría provocado tasas de explotación que habrían variado entre $24,4-26,6 \%$ en machos y $25,2-26,9 \%$ en hembras.

En términos globales, para un valor central de $\mathrm{M}=0,18$, las mortalidades por pesca globales alcanzaron en machos $\mathrm{F}=0,32$ y en hembras $\mathrm{F}=0,33$, situación equivalente a tasas de explotación en torno al $26 \%$. 


\section{Estado de explotación}

La determinación del estado de explotación de la langosta de Juan Fernández en la islas Robinson Crusoe y Santa Clara, realizado sobre la base de un valor $\mathrm{M}=0,18$ y al análisis de las mortalidades por pesca referenciales derivadas del modelo de rendimiento equilibrado de Thompson y Bell (1934), indican que durante el período analizado, la mortalidad por pesca de machos $\mathrm{F}_{\mathrm{cr}}=0,63$ y de hembras $\mathrm{F}_{\mathrm{cr}}$ $=0,81$, resultaron inferiores al valor $\mathrm{F}_{\text {máx }}$ estimado en 0,86 en machos y 1,15 en hembras, y superior a un nivel de mortalidad $\mathrm{F}_{0.1}$ estimada de 0,30 y 0,31 en machos y hembras respectivamente (Tabla 4, Fig. $3)$.

Esta situación indica que los actuales niveles de explotación resultan intensos y con tasas de explotación que reducirían la biomasa media en el largo

Tabla 2. Parámetros del patrón de explotación y mortalidad por pesca de los grupos completamente reclutados a la pesquería.

Table 2. Explotation pattern and fishing mortality parameters of the completely recruted groups of the fishery.

\begin{tabular}{|lcccc|}
\hline & \multicolumn{4}{c|}{ Parámetros vector mortalidad por pesca } \\
\hline Machos & $\mathrm{a}$ & $\mathrm{b}$ & $\mathrm{c}$ & $\mathrm{F}_{\text {cr }}$ \\
Valor central & 50,894 & 0,326 & 1,109 & 0,630 \\
Error estándar & 5,344 & 0,686 & 0,465 & 0,020 \\
Hembras & $\mathrm{a}$ & $\mathrm{b}$ & $\mathrm{c}$ & $\mathrm{F}_{\text {cr }}$ \\
Valor central & 59,782 & 0,387 & 1,102 & 0,814 \\
Error estándar & 14,316 & 0,709 & 0,440 & 0,038 \\
\hline
\end{tabular}

plazo, a niveles equilibrados cercanos a las 50 toneladas anuales totales (machos+hembras), magnitud significativamente inferior si se aplicara una tasa de explotación equivalente a $\mathrm{F}_{0.1}$, donde se alcanzaría un tamaño poblacional de aproximadamente 70 ton anuales, en el largo plazo.

En este contexto, esta condición de explotación debe ser atendida con cautela, más aún si se considera, que para especies de características biológicas similares, explotadas en otras latitudes, tasas de explotación superiores al 10\% generan serias disminuciones de los efectivos poblacionales.

La Tabla 4 y Fig. 3 muestran que para una estrategia de explotación $\mathrm{F}_{0.1}$, el valor recomendado de desembarque equivale a 10,4 y 14, 6 ton de machos $\mathrm{y}$ hembras respectivamente, cifras inferiores en un $10 \%$ respecto de los actuales niveles desembarcados. En otros términos, ante los actuales niveles de mortalidad por pesca observados, se recomienda reducir levemente los desembarques anuales en estas islas a niveles que minimicen los efectos de sobrepesca por crecimiento en el largo plazo, y que correspondan con una estrategia de explotación de $\mathrm{F}_{0.1}$ equivalente a 25 toneladas anuales.

Los resultados derivados de la talla crítica del recurso langosta se presentan en la Tabla 5, donde se aprecia que para un rango de variación de la mortalidad natural de $M=0,16-0,20$, el rendimiento de una cohorte de machos se maximiza entre $\operatorname{los} 105 \mathrm{y}$ $116 \mathrm{~mm}$ de longitud cefalotorácica, en cambio en hembras, esto ocurre entre 101 y $114 \mathrm{~mm}$ de longitud cefalotorácica.
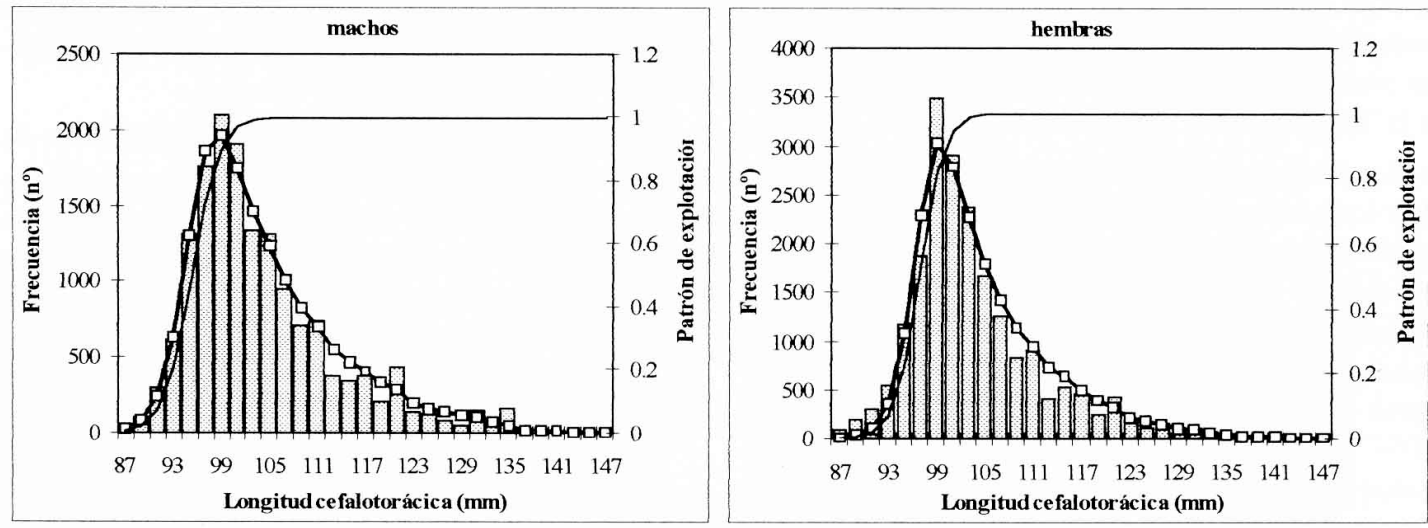

Figura 2. Frecuencia de tallas del desembarque observado y estimado, y patrón de explotación de Jasus frontalis.

Figure 2. Lenght frecuency of the observed and estimated landing, and the explotation pattern of Jasus frontalis. 
La situación anterior se ilustra en las curvas presentadas en la Fig. 4, las cuales describen el comportamiento de la pendiente de la biomasa inexplotada en función de la talla, para dos valores de $M(M=0,16$ y 0,20$)$. En este sentido, se destaca que con independencia del peso medio de los ejemplares, la talla donde la derivada de la función se hace cero, está correlacionada negativamente con la mortalidad natural, vale decir, a mayor valor de $\mathrm{M}$, menor será el estimado de la talla crítica.

Por otro lado, y para evaluar la actual talla mínima legal de captura, en relación a tallas (o edades) en las cuales se generen mayores rendimientos de pesca, se aplicó el modelo de rendimiento por recluta de Beverton y Holt (1957), para 3 distintas edades probables de primera captura (7, 9 y 11 años) y para un valor central de $\mathrm{M}=0,18$. Los resultados se indican en la Fig. 5, donde se advierte que para machos y hembras, la curva que maximiza el rendimiento unitario por sobre niveles de mortalidad por pesca de $\mathrm{F}=0,2$, corresponde al grupo de edad de 9 años, edad que efectivamente equivale con la edad crítica estimada en ambos sexos (Tabla 5).

De lo anterior se desprende que si bien en estas islas la actual talla mínima de captura resulta levemente inferior al rango de talla crítica estimada por sexo, la probable varianza que encierra el grupo de edad 9 hace que la actual medida de conservación del recurso sea adecuada y eficiente, sin evidencias de sobrepesca por crecimiento por un virtual des-
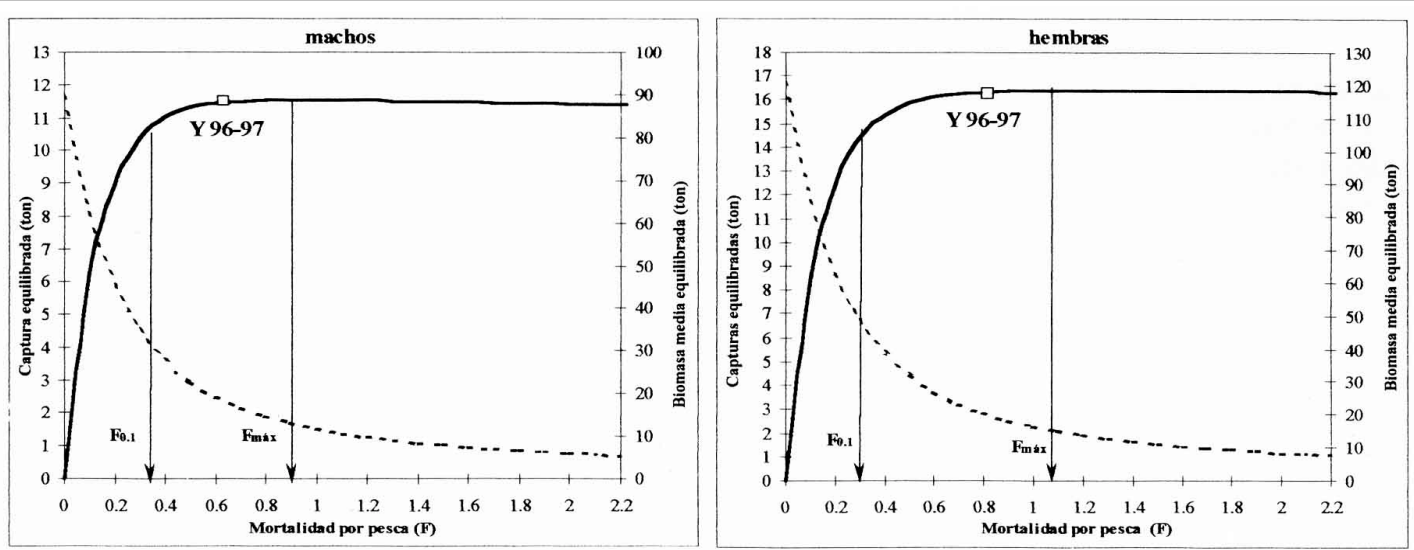

Figura 3. Capturas y biomasas medias equilibradas de largo plazo.

Figure 3. Long term equilibrated catches and biomasses means.

Tabla 3. Desembarque total, biomasas y abundancias medias totales y explotables, mortalidades por pesca globales y tasas de explotación estimadas para tres valores probables de $M$.

Table 3. Total landings, total and explotables biomass and abundance means, global fishing mortality and explotations rates estimates for three values of $M$.

\begin{tabular}{|lcccccc|}
\hline & \multicolumn{2}{c}{$\mathbf{M = 0 , 1 6}$} & \multicolumn{2}{c|}{$\mathbf{M = 0 , 1 8}$} & \multicolumn{2}{c|}{$\mathbf{M}=\mathbf{0 , 2 0}$} \\
hachos & hembras & machos & hembras & machos & hembras \\
\hline Desembarques (t) & 11,5 & 16,3 & 11,5 & 16,3 & 11,5 & 16,3 \\
Capturas $\left(\mathrm{n}^{\circ}\right.$ ) & 15.733 & 20.431 & 15.733 & 20.431 & 15.733 & 20.431 \\
Biomasa media (t) & 28,7 & 39,4 & 29,8 & 40,5 & 30,9 & 41,7 \\
Abundancia media & 46.663 & 59.785 & 48.480 & 61.552 & 50.355 & 63.249 \\
Biomasa explotable (t) & 14,5 & 17,3 & 15,0 & 17,8 & 15,5 & 18,4 \\
Abundancia explotable & 18.433 & 20.704 & 19.048 & 21.299 & 19.707 & 21.927 \\
Mortalidad por pesca global & 0,3370 & 0,3420 & 0,3245 & 0,3319 & 0,3120 & 0,3230 \\
Tasa de explotación & $26,6 \%$ & $26,9 \%$ & $25,5 \%$ & $26,0 \%$ & $24,4 \%$ & $25,2 \%$ \\
\hline
\end{tabular}


aprovechamiento en el crecimiento somático de este recurso.

Sin embargo, se debe considerar que no necesariamente una determinada talla mínima de extracción o mortalidades por pesca inferiores a las recomendadas, aseguran que la población no sufrirá reducción por pesca. En este contexto, y para recursos de un alto valor agregado como es la langosta, es posible que en el extremo, la explotación de los individuos de tallas comerciales llegue a tales niveles que el potencial reproductor presente un significativo detrimento, tendiendo positivamente hacia el fenómeno conocido como sobrepesca por reclutamiento, lo que conlleva finalmente al colapso de una pesquería.

Tabla 4. Mortalidades por pesca de referencia y capturas biológicas adecuadas (CBA).

Table 4. Reference fishing mortalities and acceptable biological catches (CBA).

\begin{tabular}{|lccc|}
\hline & \multicolumn{3}{c}{ Mortalidades por pesca de referencia } \\
Machos & $\mathbf{F}_{\mathbf{0 . 1}}$ & Fcr & Fmáx \\
\hline Valor F & 0,300 & 0,630 & 0,868 \\
Valor CBA (t) & 10,40 & 11,49 & 11,53 \\
Hembras & $\mathrm{F}_{0.1}$ & $\mathrm{Fcr}$ & Fmáx \\
\hline Valor F & 0,311 & 0,814 & 1,155 \\
Valor CBA (t) & 14,59 & 16,27 & 16,40 \\
Ambos sexos & $\mathrm{F}_{0.1}$ & Fcr & Fmáx \\
\hline Valor CBA (t) & 24,99 & 27,76 & 27,93 \\
\hline
\end{tabular}

Lamentablemente, esta situación podrá ser evaluada sólo en la medida que se conozca el grado de respuesta combinado entre reclutamiento, stock y mortalidad por pesca, situación imposible de precisar a la luz de estos resultados, pero válidos para ser tomados en cuenta al establecer medidas de conservación que tiendan a proteger una determinada fracción del stock.

\section{Análisis de variabilidad de los estimadores poblacionales}

Con el fin de proveer un rango de variabilidad de los estimados de abundancia y biomasa de langosta de Juan Fernández, se aplicó la técnica bayesiana sobre los 3 parámetros que constituyen el vector de mortalidades por pesca a saber: los parámetros a y b que configuran el patrón de explotación, y el valor que adoptó la mortalidad por pesca de los individuos completamente reclutados a la pesquería $\left(\mathrm{F}_{\mathrm{cr}}\right)$.

Los resultados de esta experiencia son mostrados en las Figs. 6 y 7, en tanto que los distintos estimadores se describen en la Tabla 6. En este contexto y conforme a lo esperado, la distribución de probabilidades de la abundancia y biomasa media presentaron un característico comportamiento normal, con valores promedios y medianos significativamente similares en cada sexo. De lo anterior se desprende que la biomasa media de machos y hembras tendría un error de $\pm 1,0$ y 0,6 ton respectivamente, en tanto que la abundancia media acusa una dispersión equivalente a \pm 1.144 machos y \pm 1.623 hembras.
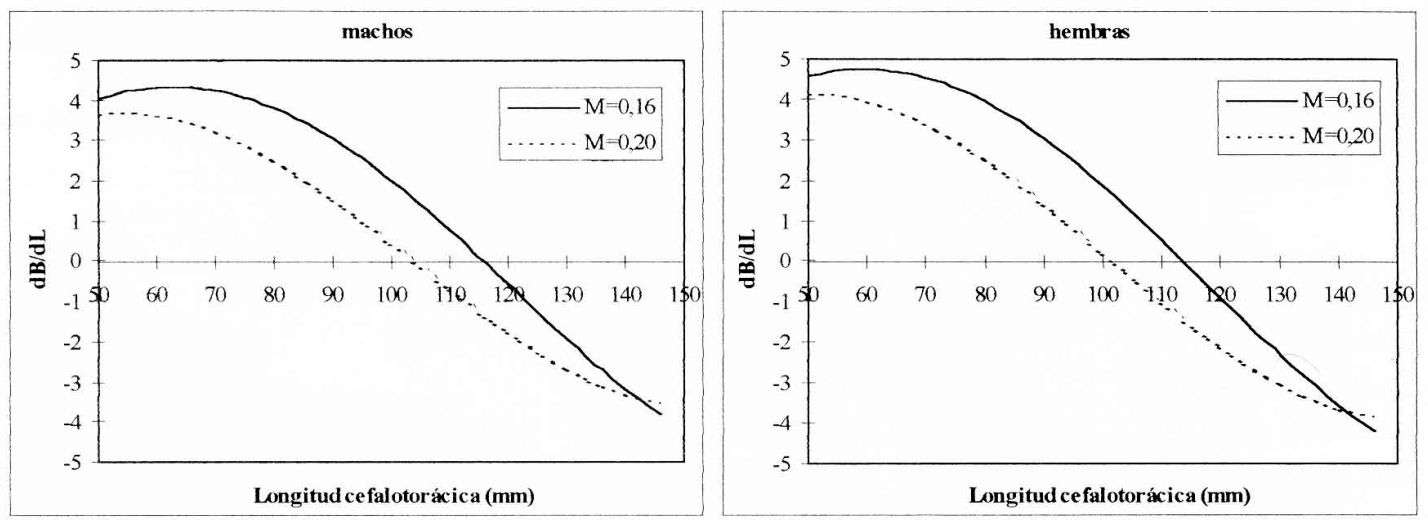

Figura 4. Derivada de la biomasa inexplotada $(\mathrm{Z}=\mathrm{M})$ respecto de la talla $\mathrm{y}$ en función de dos valores de $\mathrm{M}$.

Figure 4. Inexploted biomass derived $(\mathrm{Z}=\mathrm{M})$ in function of lenght and two values of $M$. 
Tabla 5. Talla y edad crítica en función de dos valores de M.

Table 5. Critical lenght and age in function of two values of $M$.

\begin{tabular}{|lccc|}
\hline & \multicolumn{2}{c}{ Talla crítica $(\mathbf{m m})$} & Edad crítica \\
& $\mathbf{M = 0 , 1 6}$ & $\mathbf{M = 0 , 2 0}$ & $(\mathbf{M = 0 , 1 8})$ \\
\hline Machos & 116 & 105 & 9 años \\
Hembras & 114 & 101 & 9 años \\
\hline
\end{tabular}

Tabla 6. Variabilidad de los estimadores poblacionales.

Table 6. Population parameters variability.

\begin{tabular}{|c|c|c|c|c|}
\hline & \multicolumn{2}{|c|}{ Biomasa media (ton) } & \multirow{2}{*}{$\begin{array}{r}\text { Abundancia } \\
\text { Machos }\end{array}$} & \multirow{2}{*}{$\begin{array}{l}\text { media }\left(\mathrm{n}^{0}\right) \\
\text { Hembras }\end{array}$} \\
\hline & Machos & Hembras & & \\
\hline Promedio & 29,8 & 40,5 & 48.480 & 61.552 \\
\hline Mediana & 29,8 & 40,4 & 48.527 & 61.529 \\
\hline Moda & 28,8 & 43,0 & 42.192 & 65.824 \\
\hline Error estándar & 3,7 & 5,2 & 6.195 & 8.352 \\
\hline
\end{tabular}
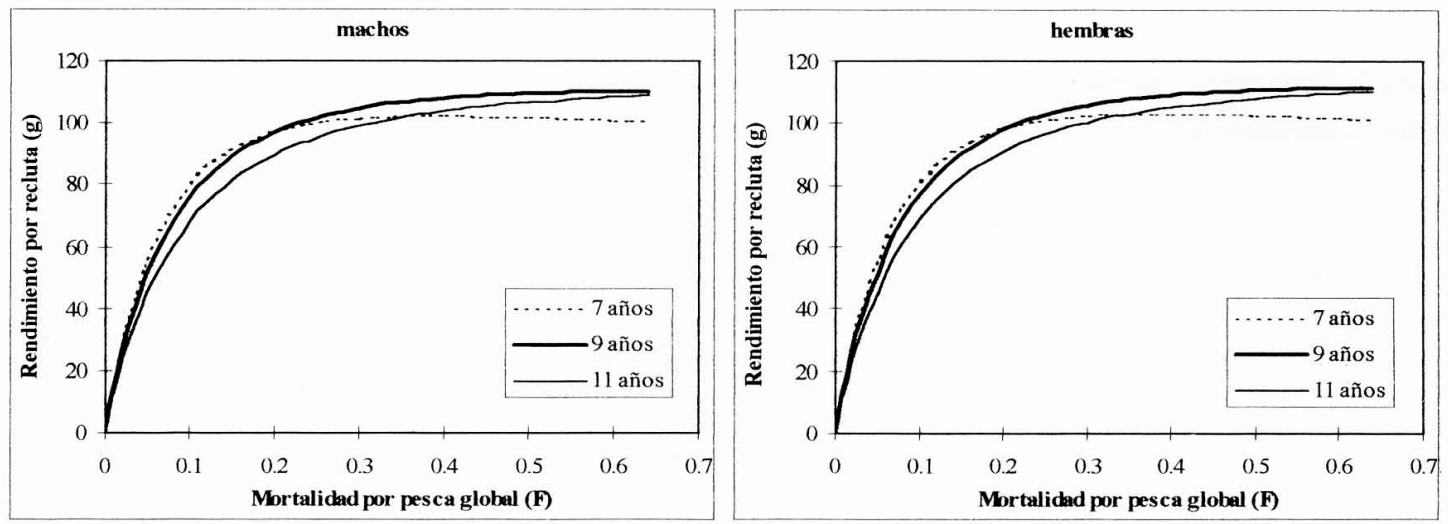

Figura 5. Curvas de rendimiento por recluta obtenidas utilizando diferentes edades de primera captura.

Figure 5. Yield per recruit curves obtained using diferents first age catch.
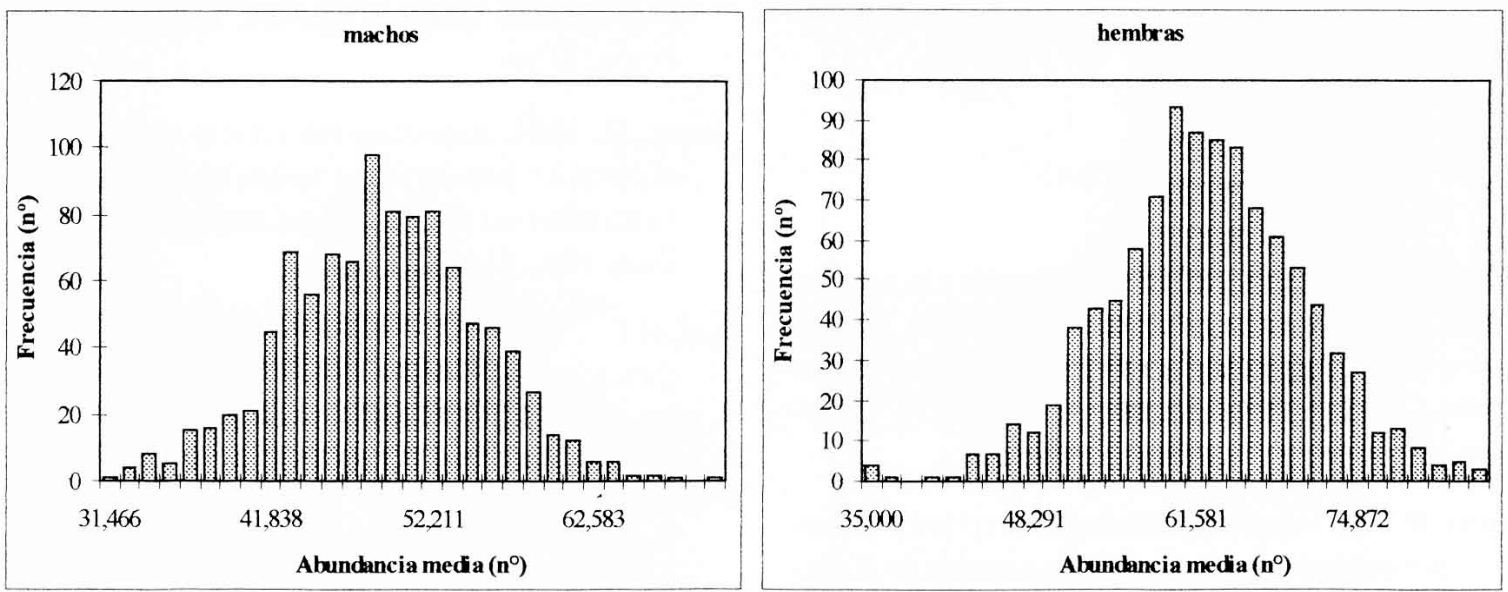

Figura 6. Variabilidad de los estimadores de abundancia de Jasus frontalis.

Figure 6. Abundance variability of Jasus frontalis. 

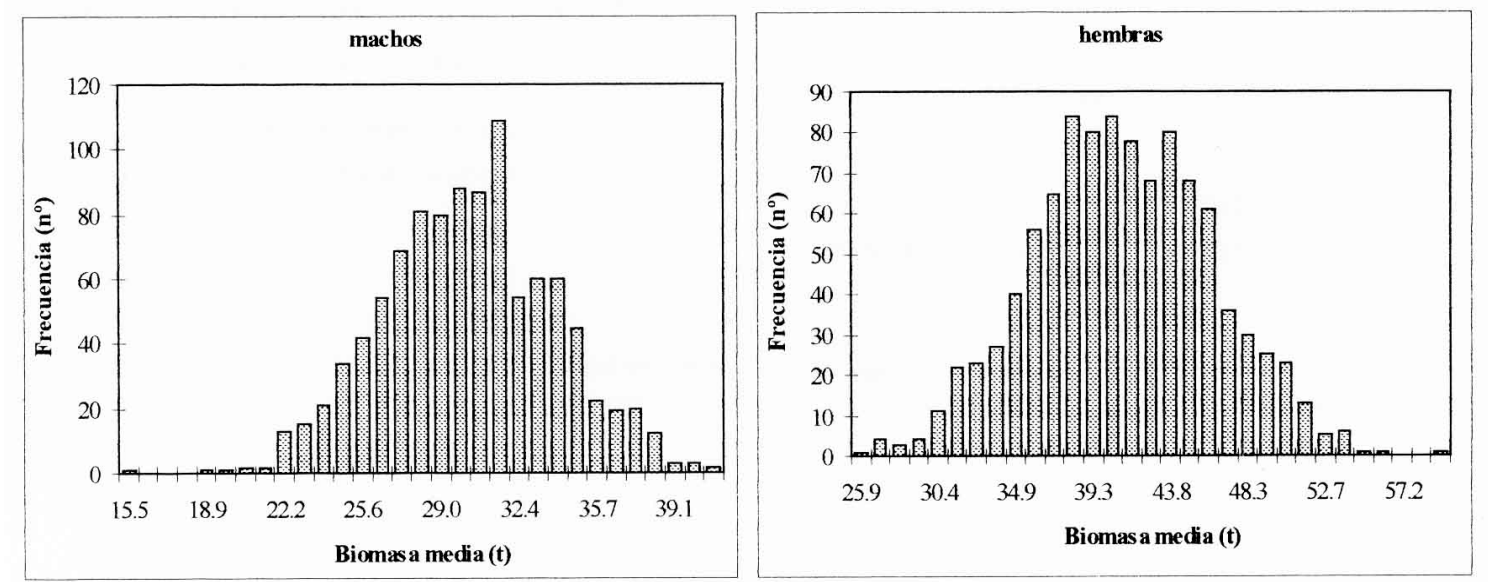

Figura 7. Variabilidad de los estimadores de biomasa de Jasus frontalis.

Figure 7. Biomass variability of the Jasus frontalis.

Finalmente, se debe destacar que, en general, el bajo error estándar de los estimadores poblacionales se tradujo en valores que no excedieron el $14 \%$ del valor central, situación que se debe exclusivamente a la bondad del modelo de evaluación empleado y a la precisión de los parámetros determinados.

El estado de situación de la langosta indica que los actuales niveles de explotación resultan intensos y con tasas de explotación que reducirían la biomasa media en el largo plazo, a niveles equilibrados cercanos a 50 ton anuales totales (machos+hembras), magnitud significativamente inferior si se implementara una tasa de explotación equivalente al punto biológico $\mathrm{F}_{0.1}$, donde se alcanzaría en equilibrio de largo plazo, un tamaño poblacional equivalente a 70 ton anuales.

\section{REFERENCIAS}

Arana, P. 1983. Estado en que se encuentra la pesquería de la langosta de Juan Fernández (Jasus frontalis). En: P. Arana (ed.). Análisis de Pesquerías Chilenas. Esc. Ciencias del Mar, UCV, Valparaíso, 77-111.

Arana, P. y R. Vega. 2000. Esfuerzo, captura y captura por unidad de esfuerzo en la pesquería de la langosta de Juan Fernández (Jasus frontalis) durante la temporada de pesca 1996-1997. Invest. Mar., Valparaíso, 28: 117-133.
Arana, P. y C. Olate. 2000. Composición de las capturas de la langosta de Juan Fernández (Jasus frontalis), durante la temporada de pesca 19961997. Invest. Mar., Valparaíso, 28: 83-115.

Baranov, F.I. 1918. On the question of the biological basis of fisheries. Nauchn. Issled. Ikhtiol. Inst. Izv., 1: 81-128.

Berverton, R.J. y S.J. Holt. 1957. On the dynamics of exploited fish population. Min. Agr. Fish and Food (UK), Fish. Investig. Ser., II, 553 pp.

Canales, C. 1994. Evaluación del stock y estimación de una captura total permisible para el recurso langostino amarillo (Cervimunida johni) en la III y IV Regiones. Informe Técnico, Subsecretaría de Pesca, 22 pp.

Jones, R. 1984. Assessing the effects of changes in explotation pattern using lenght composition data (with notes on VPA an cohort analysis). FAO Fish. Tech. Pap., 256: 1-118.

Lai, H.L. y A. Amjoun. 1993. User's guide to CASA, Catch-At-Size- Analysis, Version 1.1 for Microcomputer. Working Paper $\mathrm{N}^{\circ} 102$.

Larraín, F. 1981. Análisis histórico (1972-1979) de la pesquería de langosta (Jasus frontalis, H. Milne Edwards 1837) realizada en las islas Robinson

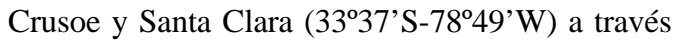
de modelos globales de producción. Tesis, Esc. Ciencias del Mar, UCV, 148 pp. 
Mac Call, A.D. 1986. Virtual population analysis (VPA) equations for non homogeneous populations, and a family of aproximations including improvements on Pope's cohort analysis. Can. J. Fish. Aquat. Sci., 43: 2406-2409.

Pauly, D. 1980. On the interrelationship between natural mortality, growth parameters, and mean environmental temperature in 175 fish stocks. J. Cons. int. Explor. Mer, 39(2): 175-192.

Pope, J.G. 1972. An investigation of the accuracy of virtual population analysis using cohort analysis. ICNAF Res. Bull., 9: 65-74.

Servicio Nacional de Pesca (Sernapesca). 1938-97. Anuarios estadísticos de pesca. Servicio Nacional de Pesca, Ministerio de Economía, Chile.

Taylor, C.C. 1958. Cod growth and temperature. J. Cons. int. Explor. Mer, 23(3): 366-370.

Thompson, W.F. y F.H. Bell. 1934. Biological statistics of the Pacific halibut fishery. 2. Effect of changes in the intensity upon total yield and yields per unit of gear. Rep. Int. Fish. (Pacific Halibut) Comm., 8: 1-49. von Bertalanffy, L. 1938. A quantitative theory of organic growth (Inquiries on growth laws II). Hum. Biol., 10: 181-213.

Yáñez, E., F. Larraín y P. Pavez. 1982. Análisis histórico (1972-1981) de la pesquería de langosta (Jasus frontalis) del archipiélago de Juan Fernández y estimación del estado actual del recurso. Informe Final Subsecretaría de Pesca, Estud. Doc., Univ. Católica Valparaíso, 4/82: 1-46.

Yáñez, E., L. Pizarro, M.A. Barbieri y O. Barra. 1985. Dinámica del stock de langosta (Jasus frontalis, H. Milne Edwards 1837) explotado en el

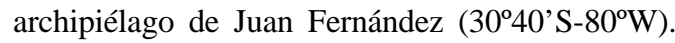
En: P. Arana (ed.). Investigaciones Marinas en el Archipiélago de Juan Fernández. Esc. Ciencias del Mar, UCV, Valparaíso, pp. 251-271.

Zuleta A. y C. Moreno. 1993. Investigación modelo y manejo pesquería recurso loco (Fase II), Informe Final Proyecto BIP 20-052-259, Universidad Austral de Chile, 41 pp. 